\title{
List of papers presented (oral and poster) at the Third Asian Photochemistry Conference (APC-2002) incorporating the Sixth Trombay Symposium on Radiation and Photochemistry
}

Only the address of the presenting author (marked with asterisk) is given in the following list.

Abbreviations: BARC: Bhabha Atomic Research Centre, IACS: Indian Association for the Cultivation of Science, IISc: Indian Institute of Science, IIT: Indian Institute of Technology, RRL: Regional Research Laboratory, SINP: Saha Institute of Nuclear Physics, TIFR: Tata Institute of Fundamental Research.

1. Isomerization in the photochemistry of aromatic hydrocarbons

Yuan Tseh Lee

Academia Sinica, Nankang, Taipei, Taiwan

2. Primary processes of spectral sensitization in photography

Keitrao Yoshihara*, Igor V Rubtsov, Kojiro Ebina, Ji-Won Oh, Shigeichi Kumazaki, Takeshi Suzumoto and Tadaaki Tani

School of Materials Science, Japan Advanced Institute of Science and Technology, Tatsunokuchi, Ishikawa 923-1292, Japan

3. Dynamics in confined systems

Kankan Bhattacharyya

Physical Chemistry Department, IACS, Kolkata 700 032, India

4. Nonlinear photochromic reaction of spirooxazine and spiropyran microcrystals induced by femtosecond laser excitation

Mototsugu Suzuki, Tsuyoshi Asahi and Hiroshi Masuhara*

Department of Applied Physics, Osaka University, Suita 565-0871, Japan

5. Luminescence studies of platinum(II) alkynyl complexes and their mixed-metal platinum(II)-copper(I) and silver(I) complexes

Vivian Wing-Wah Yam

Department of Chemistry, University of Hong Kong, Pokfulam Road, Hong Kong, P.R. China

6. Ultrafast laser-induced electron transfer: Electronic coupling, coherence effects

$F$ Willig*, C Zimmermann, S Ramakrishnan, R Eichberger and W Storck

Hahn-Meitner-Institute, Glienicker Str. 100, D-14109, Berlin, Germany

7. The studies on small free radical reactions by using time resolved FT-IR spectroscopy

Fanao Kong

Institute of Chemistry, Chinese Academy of Sciences, Beijing, P.R. China 100080

8. Designing photosystems for harvesting photons into electrons by sequential electron transfer processes: One electron reductive activations of $\alpha, \beta$ unsaturated carbonyl compounds

Ganesh Pandey

Division of Organic Chemistry (Synthesis), National Chemical Laboratory, Pune 411 008, India 
9. Photoinduced charge-separation and charge-recombination of $\mathrm{C}_{60}$-donor linked fullerenes

Osamu Ito*, Yasuyuki Araki and Mamoru Fujitsuka IMRAM and CREST, Tohoku University, Sendai, Japan

10. Scanning probe microscopy and proximity effects

Masahiko Hara

Local Spatio-Temporal functions Laboratory, Frontier Research System, RIKEN, Wako, Saitama, 3510198, Japan

11. Picosecond dynamics of photoinduced stepwise double electron transfer reaction in the 2-aminopyridine/acetic acid system

H Ishikawa, K Iwata and H Hamaguchi*

Department of Chemistry and Research Centre for Spectrochemistry, School of Science, University of Tokyo, Tokyo 113, Japan

12. Structure-reactivity of excited states quinones: Time-resolved resonance Raman (TR3) and Density Functional theoretical (DFT) study

$S$ Umapathy

Department of Inorganic and Physical Chemistry, IISc, Bangalore 560 012, India

13. Localised photochemistry with multiphoton excitation: Measuring size and stability of biomolecules

J Balaji*, K Garai and S Maiti

Department of Chemical Sciences, TIFR, Homi Bhabha Road, Mumbai 400 005, India

14. Photochemical generation and reactions of Triplet $\mathrm{Di}(9$-anthryl $)$ carbene

Hideo Tomioka*, Yoshihiro Nozaki, Eri Iwamoto and Katsuyuki Hirai

Chemistry Department for Materials, Faculty of Engineering, Mie University, Tsu, Mie 514-8507, Japan

15. Photoreactions in benzophenone/1,4-cyclohexadiene clusters

Kinichi $\mathrm{Obi}^{*}$ and Akio Kawai

Department of Chemical and Biological Sciences, Japan Women's University, 2-8-1, Mejirodai, Bunkyoku, Tokyo 112-8681, Japan

16. Theory of the electric field effect on fluorescence quenching due to electron transfer

Masanori Tachiya

National Institute of Advanced Industrial Science and Technology (AIST), Tsukuba, Ibaraki 305-8565, Japan

17. State resolved dissociation dynamics of $\mathrm{HOCl}$ near threshold

Amitabha Sinha

Department of Chemistry and Biochemistry, University of California - San Diego 9500 Gilman Drive, La Jolla, CA 92093-0314, USA

18. Application of time-resolved Fourier-transform spectroscopy to photo-induced systems: Reaction intermediates and kinetics

Yuan-Pern Lee

Department of Chemistry, National Tsing Hua University, Hsinchu 30013, Taiwan

19. Photoinduced reactions in mass-selected complexes $\mathrm{M}^{+} \mathrm{L}_{n} \quad(\mathrm{M}=$ alkaline metal atoms; $\mathrm{L}=$ organic molecules)

Shihe Yang

Department of Chemistry, Hong Kong University of Science and Technology, Clear Water Bay, Kowloon, Hong Kong 
20. Spectroscopy of cooled and trapped atoms and molecules

$B N$ Jagtap

Laser and Plasma Technology Division, BARC, Mumbai 400 085, India

21. Excited state studies of linear polyenes and related protein photoreceptors

Anil K Singh

Department of Chemistry, IIT - Bombay, Mumbai 400 076, India

22. Subpicosecond dynamics of the excited states of LDS 821 in solutions

D K Palit

Radiation Chemistry and Chemical Dynamics Division, BARC, Mumbai 400 085, India

23. Novel strategy for long-lived charge-separation

Kyung Byung Yoon

Centre for Microcrystal Assembly and Department of Chemistry, Sogang University, Seoul 121-742, Korea

24. Photodissociation dynamics of carboxylic acids: The $\mathrm{OH}$ reaction channel

P D Naik*, H P Upadhyaya, Awadhesh Kumar, D K Maity, A V Sapre and J P Mittal

Radiation Chemistry and Chemical Dynamics Division, BARC, Mumbai 400 085, India

25. Effect of alkali-metal ions on the photooxygenation of aromatic alkenes in zeolite nanocavities

Masanobu Kojima

Department of Bioscience and Biotechnology, Faculty of Agriculture, Shinshu University, 8304

Minami-minowa, Nagano 399-4598, Japan

26. Photoexcitation dynamics of porphyrin arrays as revealed by electric field effects on fluorescence

Nobuhiro Ohta*, Yuji Iwaki, Naoki Aratani and Atsuhiro Osuka

Research Institute for Electronic Science, Hokkaido University, Sapporo 060-0812, Japan

27. Photolysis of $o$-quinodimethane in room temperature solutions

Akihiko Ouchi

National Institute of Advanced Industrial Science and Technology, Tsukuba, Ibaraki 305-8565, Japan

28. Photochemical and photophysical studies of donor/acceptor-substituted butadiene liquid crystals

Suresh Das

Photochemistry Research Unit, RRL (CSIR), Thiruvananthapuram 695 019, India

29. Conformational switching and exciton interactions in hemicyanine based bichromophores

K George Thomas

Photochemistry Research Unit, RRL (CSIR), Thiruvananthapuram 695 019, India

30. Oxygen 18 separation by IRMPD

Tetsuro Majima

Institute of Scientific and Industrial Research (SANKEN), Osaka University, Mihogaoka 8-1, Ibaraki, Osaka 567-0047, Japan

31. Highly stereoselective photocycloaddition via exciplexes

Kazuhiko Mizuno

Department of Applied Chemistry, Graduate School of Engineering, Osaka Prefecture University, 1-1 Gakuen-cho, Sakai, Osaka 599-8531, Japan 
32. Singlet oxygen-superior hardware and recent advances

$J C$ Penedo*, D U Nather and E Blackwood

Edinburgh Instruments Ltd., 2 Bain Square, Kirkton Campus, Livingston, EH54 7 DQ, UK

33. Intramolecular charge transfer in micelle and cyclodextrin: Photophysics and applications for molecule recognition and chemical sensing

Yun-Bao Jiang*, Li-Rong Lin, Hong Chen and Li-Hua Ma

Department of Chemistry and the MOE Key Laboratory of Analytical Sciences, Xiamen University, Xiamen 361005, China

34. Light-emitting molecular rectangles

Kuang-Lieh Lu

Institute of Chemistry, Academia Sinica, Taipei 115, Taiwan

35. Coherent vibrational motion during excited-state intramolecular proton transfer

Re-Ming R Hsieh, Jui-Ying Lin, Charlene Su and Po-Yuan Cheng*

Department of Chemistry, National Tsing Hua University, Hsinchu, Taiwan

36. Gas phase supersonic studies of the H-bonded complexes of para-substituted phenols

Sanjay Wategaonkar

Department of Chemical Sciences, TIFR, Homi Bhabha Road, Mumbai 400 005, India

37. Interfacial electron transfer dynamics in dye sensitized $\mathrm{TiO}_{2}$ nano-particles

Hirendra N Ghosh

Radiation Chemistry and Chemical Dynamics Division, BARC, Mumbai 400 085, India

38. Photophysical and photochemical properties of 1,4-tetracenequinone in solution

Minoru Yamaji*, Takao Itoh, Shizuka Haruo and Seiji Tobita

Department of Chemistry, Gunma University, Kiryu, Gunma 376-8515, Japan

39. Exciplex parameters of N-ethyl carbazole-dimethyl terephthalate system

Sharmistha Dutta Choudhury*, Suchandra Bandyopadhyay and Samita Basu

Chemical Sciences Division, SINP,1/AF, Bidhannagar, Kolkata 700 064, India

40. Photochemistry of some carbonyl compounds in the presence of amines and titanium dioxide

Sung Sik Kim

Department of Chemistry, Chonbuk National University, Chonju 561-756, Korea

41. Laser flash photolysis studies on a novel synthesized bichromophore containing 4-methoxybenzom $[b]$ thiophene and $p$-chloroacetophenone as reacting partners

Manisankar Maiti and Tapan Ganguly*

Department of Spectroscopy, IACS, Jadavpur, Kolkata 700 032, India

42. Photophysics of phenyldibenzophosphole

Tanushree Bhattacharya and Tapan Ganguly*

Department of Spectroscopy, IACS, Jadavpur, Kolkata 700 032, India

43. Femtosecond dynamics in she $\mathrm{S}_{2}$ state of a triphenylmethane dye, malachite green

A C Bhasikuttan*, A V Sapre and T Okada

Department of Chemistry, Graduate School of Engineering Science, Osaka University, Toyonaka, Osaka, 560 8531, Japan 
44. Probing phase transitions in frozen solvents by fluorescence

VVN Ravi Kishore and N Periasamy*

Department of Chemical Sciences, TIFR, Homi Bhabha Road, Colaba, Mumbai 400 005, India

45. Intra- and inter-molecular $(2 \pi+2 \pi)$ photocycloaddition and cycloreversion of ferulic acid derivatives

Keisuke Nishimura, Hisaji Taniguchi, Akira Sugimoto and Kazuhiko Mizuno*

Department of Applied Chemistry, Graduate School of Engineering, Osaka Prefecture University, 1-1 Gakuen-cho, Sakai, Osaka 599-8531, Japan

46. Effect of solvent and interaction of metal ions with indole acids: A fluorescence study

M Rele, S Kapoor* and T Mukherjee

Radiation Chemistry and Chemical Dynamics Division, BARC, Mumbai 400 085, India

47. Photophysical properties of coumarin-152 and coumarin-481 dyes

$S \mathrm{Nad}^{*}$ and $\mathrm{HPal}$

Radiation Chemistry and Chemical Dynamics Division, BARC, Mumbai 400 085, India

48. Photophysics of $\propto$ furil: A laser flash photolysis study

A K Singh*, D K Palit and J P Mittal

Radiation Chemistry and Chemical Dynamics Division, BARC, Mumbai 400 085, India

49. Photochemistry of 3,5-dihydro-5,9b-o-benzenonaphtho[1,2c]furan-1-one

Jean John Vadakkan* and S Prathapan

Dept. of Applied Chemistry, Cochin University of Science and Technology, Kochi 682 022, India

50. Magnetic field effects on spin-correlated radical ion pair in photoinduced electron transfer

Sharmistha Dutta Choudhury, Suchandra Bandyopadhyay, Kakali Sen, Tamal Sengupta and Samita Basu*

Chemical Sciences Division, SINP, 1/AF, Bidhannagar, Kolkata 700 064, India

51. Molecular recognition of quinones. Photosensitized electron transfer reactions of rhenium(I) containing molecular rectangles with quinines

T Rajendran*, Bala Manimaran, Rong-Tang Liao, Yen-Hsiang Liu, P Thanasekaran, Ren-Jay Lin, I-Jy Chang, S Rajagopal and Kuang-Lieh Lu

School of Chemistry, Madurai Kamaraj University, Madurai 625 021, India

52. Photophysical studies on 5-hydroxyindole (5HI): Laser flash photolysis study

Suman Kalyan Pal and T Misra*

Department of Spectroscopy, IACS, Kolkata 700 032, India

53. Novel active zinc oxide species on silica for phosphorescence and photo-induced non-oxidative methane coupling

Chaskar Manohar Ganpat*, Hisao Yoshida, Yuko Kato and Tadashi Hattori

Department of Applied Chemistry, Graduate School of Engineering, Nagoya University, Furo-cho, Chikusa-ku, Nagoya-464-8603, Japan

54. $\mathrm{TiO}_{2}$ mediated photodeoxygenation of some organic compounds

$N$ Somasundaram and C Srinivasan*

School of Chemistry, Madurai Kamaraj University, Madurai 625 021, India 
55. Near-field fluorescence microspectroscopy and photothermal surface modification of poly(substituted thiophene) film

Naonori Kurokawa*, Hiroyuki Yoshikawa and Hiroshi Masuhara

Department of Applied Physics, Osaka University, 2-1 Yamada-oka, Suita, Osaka 565-0871, Japan

56. The effect of alumina on photocurrent increment in dye sensitized photoelectrochemical cells based on nanocrystalline $\mathrm{SnO}_{2}$ films

V P S Perera*, G K R Senadeera and K Tennakone

Institute of Fundamental Studies, Hantane Road, Kandy, Sri Lanka

57. $p-4 \mathrm{CuBr} .3 \mathrm{~S}\left(\mathrm{C}_{4} \mathrm{H}_{9}\right)_{2}$ : Promising material as the hole collector in solid state dye sensitized photovoltaic cells with $\mathrm{TiO}_{2}$

$G K R$ Senadeera*, D B R A De Silva, $P V V$ Jayaweera and K Tennakone

Institute of Fundamental Studies, Hantana Road, Kandy, Sri Lanka

58. Photodimerisation of enones in clay microenvironment

D Madhavan and K Pitchumani*

School of Chemistry, Madurai Kamaraj University, Madurai 625 021, India

59. Asymmetric induction in photochemical reactions: Photochemistry of $\alpha$-oxomides included in zeolites

N Arunkumar* and V Ramamurthy

Department of Chemistry, Tulane University, New Orleans, LA 70118, USA

60. Efficient electron injection from TICT state of 7-diethyl amino coumarin 3carboxylic acid (D-1421) dye to $\mathrm{TiO}_{2}$ nanoparticle

G Ramakrishna* and H N Ghosh

Radiation Chemistry and Chemical Dynamics Division, BARC, Mumbai 400 085, India

61. Electron transfer dynamics in di-bromo fluorescin (DBF) sensitized $\mathrm{TiO}_{2}$ nanoparticle surface: Effect of solvent polarity and free energy $(-\Delta G)$ of the reaction

G Ramakrishna*, Amit Das and H N Ghosh

Radiation Chemistry and Chemical Dynamics Division, BARC, Mumbai 400 085, India

62. Effect of particle size on reactivity of quantum size $\mathrm{ZnO}$ nanoparticles

G Ramakrishna* and H N Ghosh

Radiation Chemistry and Chemical Dynamics Division, BARC, Mumbai 400 085, India

63. Fluorescence anisotropy decay in polymar-surfactant aggregates

Sobhan Sen, Dipankar Sukul, Partha Dutta and Kankan Bhattacharyya*

Physical Chemistry Department, IACS, Jadavpur, Kolkata 700 032, India

64. Dual emission of 4-(N,N-dimethylamino) cinnamaldehyde in cationic, anionic and non-ionic micelle

Subhasis Panja*, Papia Chowdhury and Sankar Chakravorti

Department of Spectroscopy, IACS, Jadavpur, Kolkata 700 032, India

65. Slow solvation dynamics of dimethylformamide in a nanocavity. 4-aminophthalimide in $\beta$-cyclodextrin

Sobhan Sen, Dipankar Sukul, Partha Dutta and Kankan Bhattacharyya*

Physical Chemistry Department, IACS, Jadavpur, Kolkata 700 032, India 
66. Dynamics of nile red included in $\beta$ and $\gamma$ cyclodextrins by time resolved fluorescence spectroscopy

MS Kulkarni* and Ranjan Das

Department of Chemical Sciences, TIFR, Colaba, Mumbai 400 005, India

67. Photophysical and lasing characteristics of dye doped polymer active medium

Alok Ray*, S K Nayak, Sucharita Sinha and K Dasgupta

Laser and Plasma Technology Division, BARC, Mumbai 400 085, India

68. Synthesis and properties of new electroluminescent polymers: Possessing 8-hydroxyquinoline metal complex chromophores

Sang Woo Kim* and Sang Chul Shim

Photochemistry Laboratory, Center for Molecular Design and Synthesis, Department of Chemistry, School of Molecular Science-BK21, Korea Advanced Institute of Science and Technology 373-1, Kusung-Dong, Yusung-Gu, Taejon 305-701, Korea (ROK)

69. Design of chiral nematic glasses using hydrogen bonding interactions V Ajay Mallia*, $P$ K Sudhadevi Antharjanam and Suresh Das

Photochemistry Research Unit, RRL (CSIR), Thiruvanantapuram 695 019, India

70. Photo-induced host guest electron transfer in a $\mathrm{C}_{60}$-fullerene containing phosphate glass

$R$ Debnath* and $R$ Sahoo

Central Glass and Ceramic Research Institute, Kolkata 700 032, India

71. Photoinduced electron transfer from excited biphenyldiols and calixarenes to chloroalkanes

J Mohanty*, H Pal, S Nayak, S Chattopadhyaya and A V Sapre

Radiation Chemistry and Chemical Dynamics Division, BARC, Mumbai 400 085, India

72. PET between coumarin dyes and aromatic amines in micelle: An example of marcus inverted region

S Nath*, M Kumbhakar, H Pal, T Mukherjee and A V Sapre

Radiation Chemistry and Chemical Dynamics Division, BARC, Mumbai 400 085, India

73. Fluorescence resonance energy transfer and diffusive dynamics of a flexible polymer chain

T Bandyopadhyaya* and $S K$ Ghosh

Radiation Chemistry and Chemical Dynamics Division, BARC, Mumbai 400 085, India

74. Evaluation of some fluorescence probes on their applicability in monitoring polymerization processes

Tincy Lis Thomas and A K Mishra*

Department of Physics, IIT - Madras, Chennai 600 036, India

75. Slowing down of ionic solvation time correlation function due to cage effect

CN Patra and S K Ghosh*

Radiation Chemistry and Chemical Dynamics Division, BARC, Mumbai 400 085, India

76. Polarized Raman scattering studies of orientational order of a liquid crystal sample (PCH5)

$S$ Kundu*, $P$ Sett, $S K$ Roy and $P$ K Mallick

Department of Spectroscopy, IACS, Jadavpur, Kolkata 700 032, India 
77. Photo-EPR and photoacoustic spectroscopic investigations of $\mathrm{Nd}^{3+}, \mathrm{Tb}^{3+}$ and $\mathrm{Er}^{3+}$ doped PVA films under insitu copper vapor laser illumination Mithlesh Kumar*, Y Babu, A R Dhobale, R M Kadam and M D Sastry Radiochemistry Division, BARC, Mumbai 400 085, India

78. Laser flash photolysis study on the reaction of benzil with amines Abha Semwal, $A K$ Singh and $D K$ Palit $^{*}$

Radiation Chemistry and Chemical Dynamics Division, BARC, Mumbai 400 085, India

79. Dissociative electron transfer between aromatic amines and chloroalkanes

$S$ Nath* and A V Sapre

Radiation Chemistry and Chemical Dynamics Division, BARC, Mumbai 400 085, India

80. Photophysical and photochemical studies of indolic stilbenoids

Prasanta K Hota and Anil K Singh*

Department of Chemistry, IIT - Bombay, Powai, Mumbai 400 076, India

81. Photophysics and photochemistry of melatonin

H S Mahal ${ }^{*}$, S Nath, D K Palit and T Mukherjee

Radiation Chemistry and Chemical Dynamics Division, BARC, Mumbai 400 085, India

82. Nitroaryl-based photoactivation of immunoglobulin

Prashat Khade and Anil K Singh*

Department of Chemistry, IIT - Bombay, Powai, Mumbai 400 076, India

83. Bacteriorhodopsin analogues based on diphenylpolyene chromophores

$D$ Manjula and Anil K Singh*

Department of Chemistry, IIT - Bombay, Powai, Mumbai 400 076, India

84. Photochemistry of bridged mixed enones: An exploratory study on route-selectivity

Saswati Lahiri*, Dwijendra Lal Maji and Mithu Chanda

Department of Organic Chemistry, IACS, Kolkata 700 032, India

85. Observation of coherent effects in pump probe experiments on ultrafast time scale and their control

$R$ Justin Rajesh and Prem B Bisht*

Department of Physics, IIT - Madras, Chennai 600 036, India

86. Influencing radiative rates of a fluophore: Fluorescence anisotropy and morphology dependent resonances

$P$ Sandeep and $P$ B Bisht*

Department of Physics, IIT - Madras, Chennai 600 036, India

87. Second order optical nonlinearity of donor-acceptor substituted phosphine-imine chromophores

Anu Krishnan, K Raghuraman, N P Kishore, Kattesh V Katti and Puspendu K Das*

Department of Inorganic and Physical Chemistry, IISc, Bangalore 560 012, India

88. Surface enhanced Raman scattering studies of triazole derivatives

Suzy Thomas, S Kapoor*, V Sugandhi, R D'Cunha and T Mukherjee

Radiation Chemistry and Chemical Dynamics Division, BARC, Mumbai 400 085, India 
89. Maximization of yield of ${ }^{13} \mathrm{C}$ isotope by multiphoton dissociation of freon-22 for macroscopic production of ${ }^{13} \mathrm{C}$ isotope using high average power tea $\mathrm{CO}_{2}$ laser Manoj Kumar, Anant Deshpande*, Chintan Gupta, A K Biswas and A K Nath Centre for Advanced Technology, Indore 452 013, India

90. An efficient product separator and collector system for molecular laser isotope separation process

$V$ Parthasarathy*, A K Nayak and S K Sarkar

Laser and Plasma Technology Division, BARC, Mumbai 400 085, India

91. On the observation of frozen light in a sample of two level atom interacting with a sqeezed reservoir

Amitabh Joshi

Laser and Plasma Technology Division, BARC, Mumbai 400 085, India

92. A free radical spectrum attributable to the triplet-triplet transition of $\mathrm{HCN}$

$R$ Venkatasubramanian* and S L N G Krishnamachari

Spectroscopy Division, BARC, Mumbai 400 085, India

93. Laser photodissociation dynamics of hydroxyacetone: Observation of nascent $\mathrm{OH}$ by laser induced fluorescence

P K Chowdhury*, H P Upadhyaya, P D Naik and J P Mittal

Radiation Chemistry and Chemical Dynamics Division, BARC, Mumbai 400 085, India

94. Quantum yield and dissociation dynamics of $\mathrm{H}$ atom formation in 193.3 and 121.6 photodissociation of acetylene $\left(\mathrm{C}_{2} \mathrm{H}_{2}\right)$

$R K$ Vatsa, K S Lee, K H Jung, A Lauter, H-R Volpp* and J Wolfrum

95. Dynamics of $\mathrm{OH}$ formation in the photodissociation of pyruvic acid at $193 \mathrm{~nm}$

S Dhanya*, R D Saini, H P Upadhyaya, Awadhesh Kumar and P D Naik

Radiation Chemistry and Chemical Dynamics Division, BARC, Mumbai 400 085, India

96. Ultrafast dynamics of nonbornene and norbirnadiene excited at $200 \mathrm{~nm}$

K K Pushpa*, W Fuss, W E Schmidt and S A Trushin

Radiation Chemistry and Chemical Dynamics Division, BARC, Mumbai 400 085, India

97. Photodissociation dynamics of propiolic acid at $193 \mathrm{~nm}$ employing laser induced fluorescence technique

Awadhesh Kumar*, H P Upadhyaya, P D Naik, A V Sapre and J P Mittal

Radiation Chemistry and Chemical Dynamics Division, BARC, Mumbai 400 085, India

98. Hydrogen bonded structure of tetrahydroisoquinoline-water clusters in supersonic jet

Nikhil Guchhait

Department of Chemistry, Visva-Bharati, Santiniketan 731 235, India

99. Water clusters of aminophenol and hydroquinone

P S Meenakshi*, Nandita Biswas and Sanjay Wategaonkar

Department of Chemical Sciences, TIFR, Colaba, Mumbai 400 005, India

100. REMPI and hole burning spectroscopy of hydroquinone dimer

P S Meenakshi, Nandita Biswas and Sanjay Wategaonkar*

Department of Chemical Sciences, TIFR, Colaba, Mumbai 400 005, India 
101. Photodissociation dynamics of $\mathrm{CH}_{2} \mathrm{ICl}$ and $\mathrm{C}_{6} \mathrm{H}_{4} \mathrm{I}\left(\mathrm{CH}_{2} \mathrm{Cl}\right)$ at 222, 236, 266, 280, and $304 \mathrm{~nm}$

Dulal Senapati and Puspendu K Das*

Department of Inorganic and Physical Chemistry, IISc, Bangalore 560 012, India

102. Excimer formation in the mixed dimers of aromatic hydrocarbons: A laserinduced fluorescence study in supersonic jet

Aloke Das*, K K Mahato and Tapas Chakraborty

Department of Chemistry, IIT, Kanpur 208 016, India

103. Theory of non-polar solvation dynamics: effect of attractive and repulsive interactions

NChoudhury* and S K Ghosh

Radiation Chemistry and Chemical Dynamics Division, BARC, Mumbai 400 085, India

104. Photooxidation of curcumin in presence of peroxydisulphate in aqueousacetonitrile medium

Middulla Sudha Swaraga* and Mundra Adinarayana

Department of Chemistry, Osmania University, Hyderabad 500 007, India

105. Kinetics and mechanism of protection of thymine from sulphate radical anion by caffeic acid under anoxic conditions

Mundra Adinarayana* and Middulla Sudha Swaraga

Department of Chemistry, Osmania University, Hyderabad 500 007, India

106. Effect of solvent on the photophysical properties of substituted curcumins

A Barik*, K I Priyadarsini, D K Palit and Hari Mohan

Radiation Chemistry and Chemical Dynamics Division, BARC, Mumbai 400 085, India

107. Ligand tuning of luminescence of rhenium(I) - based molecular rectangles

Bala Manimaran, $T$ Rajendran, Rong-Tang Liao, Ren-Jay Lin, I-Jy Chang, S Rajagopal and Kuang-Lieh Lu*

Institute of Chemistry, Academica Sinica, Taipei, 115 Taiwan

108. Synthesis and luminescence quenching studies of chromiun(III) complexes with $\mathrm{N}$ and $\mathrm{O}$ donor ligands

$S$ Balasubramanian*, $N$ Senthil Kumar and T Shanmuga Priya

Dept. of Inorganic Chemistry, A C College Campus, University of Madras, Chennai 600 025, India

109. Conformational studies on gramicidin-A in cationic micellar media by fluorescence spectroscopy

$J$ Shobini and A KMishra*

Department of Chemistry, IIT - Madras, Chennai 600376 India

110. Photoinduced proton transfer in 1-hydroxyl 2-naphthoic acid: Experimental and theoretical investigations

H Mishra*, S Maheshwari, N Sathyamurthy and H B Tripathy

Photophysics Laboratory, Physics Department, Kumaoun University, Nainital 263 001, India

111. Edge excitation red shift and charge transfer study of 8-methoxyquinoline in polymer matrices

MS Mehata*, H C Joshi and H B Tripathi

Photophysics Laboratory, Physics Department, Kumaoun University, Nainital 263 001, India 
112. Comparative studies of diffusion and migration effects on excitation energy transfer among dye pairs in different media

VMishra*, H Mishra and T Pant

Photophysics Lab, Physics Department, Kumaoun University, Nainital 263 001, India

113. Studies on uranyl ion induced photodegradation

$S K$ Rakshit, $M K$ Sureshkumar, D B Naik and K Kishore*

Applied Chemistry Division, BARC, Mumbai 400 085, India

114. Peripherally coordinated tetra ruthenated porphyrin: Photophysical investigation

$S$ Tamijselvy, $R$ Venkatesan*, T M Rajendiran and P Sambasiva Rao

Department of Chemistry, Pondicherry University, R V Nagar, Kalapet, Pondicherry 605 014, India

115. Time resolved laser induced fluorescence in nuclear fuel cycle

G K Bhowmick*, K Dasgupta, R C Bapna, $S$ K Sarkar and A Ramanujam

Laser and Plasma Technology Division, BARC, Mumbai 400 0085, India

116. Photochemistry of volatile uranium compounds

Sisir K Sarkar

Laser and Plasma Technology Division, BARC, Mumbai 400 085, India

117. Aggregation of amyloid beta peptide by fluorescence correlation spectroscopy

P Sengupta, K Garai, D J Callawan and S Maiti*

Department of Chemical Sciences, TIFR, Mumbai 400 005, India

118. Multiphoton excitation spectroscopy of biomolecules

K Garai, J Balaji and S Maiti*

Department of Chemical Sciences, TIFR, Mumbai 400 005, India

\section{Radiation Chemistry}

1. Observation of transient species in high temperature and supercritical water by pulse radiolysis

Yosuke Katsumura*, Guozhong Wu, Mingzhang Lin, Yusa Muroya, Timomi Morioka and Hisaki Kudoh

Nuclear Engineering Research Lab, School of Engineering, University of Tokyo, 2-22 Shirakata Shirane, Tokai-mura, Ibaragi Prefecture, 319-1188, Japan

2. Improvement of laser-synchronize subpicosecond pulse radiolysis and their application to geminate recombination, polymers and nanotechnology

S Tagawa*, Y Yoshida, T Kozawa, A Saeki and Shu Seki

Institute of Scientific and Industrial Research, Osaka University, 8-1, Mihogaoka, Ibaraki, Osaka, 567 0047, Japan

3. Laser driven electron accelerator at Waseda University

Masakazu Washio*, Shigeru Kashiwagi, Yoshimasa Hama, Ryunosuke Kuroda

Takashi Oshima, Junji Urakawa and X J Wang

Waseda University, 3-4-1 Okubo, Shinjuku-ku, Tokyo 169-8555, Japan

4. Radiation chemistry in model membrane system

S Adhikari

Radiation Chemistry and Chemical Dynamics Division, BARC, Mumbai 400 085, India 
5. Study of alkane radical cations in irradiated solutions by time resolved magnetic field effects

VI Borovkov, VA Bagryansky, I V Eletskikh and Yu N Molin*

Institute of Chemical Kinetics and Combustion, 630090 Novosibirsk, Russia

6. Recent progress on radiation technology applications for material processing in India Sunil Sabharwal

Radiation Technology Development Section, BARC, Mumbai 400 085, India

7. Radiation chemistry and radiation processed foods

Arun Sharma

Food Technology Division, BARC, Mumbai 400 085, India

8. Free radical reactions and antioxidant activity of curcumin and its derivatives

KIndira Priyadarsini

Radiation Chemistry and Chemical Dynamics Division, BARC, Mumbai 400 085, India

9. Redox chemistry of arylazonapthol dyes: A radiation chemical study

$K$ K Sharma and B S M Rao*

National Centre for Free Radical Research, Dept. of Chemistry, University of Pune, Pune 411 007, India

10. Reactions of oxide radical ion $\left(\mathrm{O}^{-}\right)$with substituted pyrimidines

P Manoj, H Mohan, T L Luke, V M Manoj, J P Mittal and C T Aravindakumar*

School of Chemical Sciences, Mahatma Gandhi University, Kottayam 686 560, India

11. Fenton Chemistry of 1,3-dimethyluracil

JA Theruvathu, CT Aravindakumar*, Roman Flyunt, Justus von Sonntag and Clemens von Sonntag

School of Chemical Sciences, Mahatma Gandhi University, Kottayam 686 560, India

12. Reactions of $\mathrm{OH}$ and other oxidizing radicals with tryptophol

G H Naik, KI Privadarsini* and Hari Mohan

Radiation Chemistry and Chemical Dynamics Division, BARC, Mumbai 400 085, India

13. The oxidation reactions of biphenyl dione

M Rele, S Adhikari*, G P Kalena, S Chattopadhyaya and T Mukherjee

Radiation Chemistry and Chemical Dynamics Division, BARC, Mumbai 400 085, India

14. Hole transfer in DNA

Tetsuro Majima

The Institute of Scientific and Industrial Research (SANKEN), Osaka University, Mihogaoka 8-1, Ibaraki, Osaka 567-0047, Japan

15. Radiation chemical study of DNA model systems

T S Singh, S Geeta, P O'Neill and B S M Rao*

National Centre for Free Radical Research, Dept. of Chemistry, University of Pune, Pune 411 007, India

16. Radiation induced DNA damages in Escherichia coli K12 cells in the presence of copper(II) ions and its complexes: A fluorimetric study

S Selvaraj*, D Mishra, A Chakraborty, A Saha and K Chabita

Inter-University Consortium for DAE Facilities, Sector III, Block LB, Plot 8, Bidhannagar, Kolkata 700 098, India 
17. Tempo and tempol protect DNA from $\gamma$-induced damage in aerated medium

$K$ Chabita* and P C Mandal

Chemical Sciences Division, Saha Institute of Nuclear Physics, Bidhannagar, Kolkata 700 064, India

18. Kinetics of DNA single strand break rejoining in gamma irradiated human eripheral blood leucocytes

$H N$ Bhilwade, $V$ Sonawane, R Rajagopalan and $R$ C Chaubey*

Cell Biology Division, BARC, Mumbai 400 085, India

19. Effect of gamma radiation and photolysis on aminopeptidase

V K Jamadar, S N Jamadar, Hari Mohan, S P Dandekar and P Harikumar*

Food Technology Division, BARC, Mumbai 400 085, India

20. Radiation induced polymerization and gel formation characteristics of N-hydroxy methyl acrylamide in aqueous solution

Anjali Acharya*, H Mohan and S Sabharwal

Radiation Technology Development Section, BARC, Mumbai 400 085, India

21. Radiolytic reduction of poly (para-sodium styrene sulphonate) of different degrees of polymerization in aqueous solution

Y K Bhardwaj*, H Mohan and S Sabharwal

Radiation Technology Development Section, BARC, Mumbai 400 085, India

22. Preparation of low molecular weight chitosan by a combination of chemical treatment and $\mathrm{Co}^{60}$ gamma radiation.....SP Ramnani*, $C V$ Chaudhari, $K P$ Rawat and S Sabharwal

Radiation Technology Development Section, BARC, Mumbai 400 085, India

23. The mechanical properties and the chemical structure for polyethylene irradiated with heavy ion-beams

Toshitaka Oka*, Hidenori Kanbe, Fumio Yatagai, Yoshimasa Hama and Fumio Yatagai Advanced Research Institute for Science \& Engineering, Waseda University, 3-4-1-, Okuba, Shinju-ku, Tokyo 169-8555, Japan

24. High energy $\mathrm{C}^{+}$ion induced modifications in viton and polystyrene

$B$ Ghade $i^{*}$,V Chakraborty, A Datta and A Saha

Inter-University Consortium for DAE Facilities, Calcutta Centre, Sector III, Block LB, Plot 8 , Bidhannagar, Kolkata 700 098, India

25. Extraction of plutonium using radiation grafted 2-ethylhexyl phosphonic acid mono2-ethylhexyl ester on vinyl copolymer

C S Kedari, M Kumar*, S S Pandit and U Jambunathan

Radiation Chemistry and Chemical Dynamics Division, BARC, Mumbai 400 085, India

26. Study of gamma radiolytic formation of mixed aqueous $\mathrm{Ag} / \mathrm{Tl}$ sols

Manmohan Kumar

Radiation Chemistry and Chemical Dynamics Division, BARC, Mumbai 400 085, India

27. Radiolytic and laser induced study on Ag metal nanoparticles in viscous media M Rele, S Kapoor, D K Palit and T Mukherjee*

Radiation Chemistry and Chemical Dynamics Division, BARC, Mumbai 400 085, India 
28. Transient and silver clusters in methanol

Ghasiram Dey*, Mehran Mostafavi and Jacqueline Belloni

Applied Chemistry Division, BARC, Mumbai 400 085, India

29. Degradation studies on di(2-ethylhexyl)isobutyramide (D2EHIBA): A promising extractant for the reprocessing of Th based fuels

PN Phatak*, A Subramanian, S Gamre and V K Manchanda

Radiochemistry Division, BARC, Mumbai 400 085, India

30. Effect of $\gamma$-exposure on retention of recoil ${ }^{56} \mathrm{Mn}$ in permanganates

S P Mishra* and Vijaya

Nuclear and Radiochemistry Laboratory, Department of Chemistry, Banaras Hindu University, Varanasi 221005 , India

31. Intramolecular proton transfer in water dimer on ionization

$T$ K Ghanty* and $S$ K Ghosh

Radiation Chemistry and Chemical Dynamics Division, BARC, Mumbai 400 085, India

32. Structure, bonding and spectra of two center three-electron bonded sulfur centred cations: An ab initio molecular orbital study

Dilip K Maity

Radiation Chemistry and Chemical Dynamics Division, BARC, Mumbai 400 085, India

33. Reactions of methyl viologen dication $\left(\mathrm{MV}^{2+}\right)$ with $\mathrm{H}$ atom in aqueous solution: Mechanism derived from pulse radiolysis measurements and ab initio calculations

T N Das*, T Ghanty and H Pal

Radiation Chemistry and Chemical Dynamics Division, BARC, Mumbai 400 085, India

34. Investigation on the triplet excited states and radical intermediates formed in electron pulse radiolysis of amino and dimethyl amino derivatives of benzophenone

A K Singh*, D K Palit, T Mukherjee and J P Mittal

Radiation Chemistry and Chemical Dynamics Division, BARC, Mumbai 400 085, India

35. Reactions of hydrated electrons with triazine derivatives in aqueous medium

$R$ Varghese, $H$ Mohan, TA Jacob, $P$ Manoj, VM Manoj, JP Mittal and CT Aravindakumar*

School of Chemical Sciences, Mahatma Gandhi University, Kottayam 686 560, India

36. Free radical induced oxidation and reduction of 1-arylazo-2-naphthol dyes K K Sharma, B S M Rao*, H Mohan, J P Mittal, J Oakes and P O'Neill

National Centre for Free Radical Research, Dept. of Chemistry, University of Pune, Pune 411 007, India

37. Reactions of radiolytically generated oxidizing species with vinyl benzyl trimethyl ammonium chloride (VBT) in aqueous solution

Virendra Kumar*, Y K Bhardwaj, S Sabharwal and Hari Mohan

Radiation Technology Development Section, BARC, Mumbai 400 085, India

38. Pulse radiolysis study on the redox reactions of basic fuschin, an organic dye

SN Guha

Radiation Chemistry and Chemical Dynamics Division, BARC, Mumbai 400 085, India 
39. Nano-wire formation in polymer thin films by high energy ion beams Shu Seki*, Y Yoshida, S Tagawa, M Sugimoto, N Morishita and S Tanaka

The Institute of Scientific and Industrial Research, Osaka University, 8-1, Mihogaoka, Ibaraki, Osaka, 567 0047, Japan

40. Dose rate effects on radiolytic synthesis of gold-platinum clusters

H Remita* and J Belloni

Laboratoire de Chimie Physique, Umr 8000, Bât 349, Université-Paris-Sud, 91405 Orsay, France

41. Platinum carbonyl clusters: Redox kinetics and applications

H Remita*, M Treguer, P Pernut and J Belloni

Laboratoire de Chimie Physique, Umr 8000, Bât 349,

-Paris-Sud, 91405 Orsay, France

42. Pule radiolysis of 3-pyridine methanol and 3-pyridine carboxyaldehyde in aqueous solution

D B Naik, A D Belapurkar and K Kishore*

Applied Chemistry Division, BARC, Mumbai 400 085, India 
Previous Asian Photochemistry Conferences (APC):

$\begin{array}{lll}\text { (1) Hong Kong } 1997 & \text { (2) Taejon, Korea } 1999\end{array}$

(3) Mumbai 2002

Next Asian Photochemistry Conference: Taipei, Taiwan 2004

Previous Trombay Symposia on Radiation \& Photochemistry (TSRP) held at Bhabha Atomic Research Centre, Trombay, Mumbai
(1) 1992 (2) 1994
(3) 1996
(4) 1998
(5) 2000

(6) 2002 (incorporated in APC-2002)

Next TSRP at BARC, Mumbai during January 7-12, 2004 